\section{Evaluation of an In vitro Approach to the Prediction of In vivo Effects on Multidrug Resistance in Human Hepatoma Cells}

Kosuke Inamura ${ }^{1}$, Kazumi Emoto $^{1}$, Hideaki Ichihara ${ }^{1}$, Kohei Sasaki ${ }^{2}$, Takuya Iwasa ${ }^{2}$, Rie Kojima ${ }^{2}$, Masaaki Kawabe ${ }^{2}$, Yuji Komizu ${ }^{1}$, Yoko Matsumoto ${ }^{1}$ and Taku Matsushita ${ }^{1^{*}}$

${ }^{1}$ Division of Applied Life Science, Graduate School of Engineering, Sojo University, Japan

${ }^{2}$ Central Research Laboratory, Japan Vilene Company Ltd, Japan

${ }^{*}$ Corresponding author: Taku Matsushita, Division of Applied Life Science, Graduate School of Engineering, Sojo University, 4-22-1 Ikeda, Nishi-ku, Kumamoto 860-0082, Japan, Tel: +81 963263973; Fax: +81 963231331; E-mail: matusita@life.sojo-u.ac.jp

Received date: August 17, 2018; Accepted date: September 01, 2018; Published date: September 10, 2018

Copyright: $\bigcirc 2018$ Inamura K, et al. This is an open-access article distributed under the terms of the Creative Commons Attribution License, which permits unrestricted use, distribution, and reproduction in any medium, provided the original author and source are credited.

\section{Abstract}

Objective: Multidrug resistance poses a major challenge to effective cancer chemotherapy. The present study evaluated whether a cell-based in vitro assay could provide a relevant screening system for agents with the potential to overcome cancer cell drug resistance.

Methods: We used a drug known to overcome doxorubicin resistance (verapamil) as a model compound and investigated its effects in vitro using three-dimensional Cellbed cultures of human hepatoma HepG2 cells, and in vivo using a HepG2 xenograft mouse model. In addition, we examined the expression of multidrug resistance protein 1 in vitro and in vivo using immunostaining.

Results: The half-maximal inhibitory concentration $\left(\mathrm{IC}_{50}\right)$ of doxorubicin was higher for HepG2 Cellbed cultures than in those cultured as a two-dimensional monolayer; these $\mathrm{IC}_{50}$ values were reduced by verapamil. Verapamil inhibited doxorubicin efflux from HepG2 Cellbed cultures. Doxorubicin resistance was also reduced by verapamil in vivo.

Conclusion: The three-dimensional Cellbed culture system could provide a promising cell-based in vitro assay system for the screening of agents that can overcome hepatic cancer cell drug resistance in vivo.

Keywords: Multidrug resistance; 3D culture; Silicate fiber scaffold; Cellbed; Human hepatoma; MDR1; Doxorubicin; Verapamil

\section{Introduction}

One of the major obstacles in the chemotherapeutic treatment of solid tumors is intrinsic or acquired multidrug resistance (MDR) [1]. MDR is associated with increased drug efflux from cancer cells, and is mediated by certain proteins such as P-glycoprotein (also known as MDR1) and MDR-associated protein [2]. MDR1 is primarily expressed in epithelial cells such as those of the small intestine, liver, kidney, and blood-brain barrier [3].

There is evidence suggesting that the use of small-molecule compounds that competitively inhibit drug transport by MDR1 is an effective strategy for overcoming MDR in cancer [4]. Various compounds including cyclosporine A, diltiazem, FK-506, and verapamil (VRP)-a calcium channel blocker-inhibited active drug efflux and restored the sensitivity of MDR cells to anticancer agents [5-9].

Cancer cell-based assay systems that reflect the in vivo MDR characteristics of the cells can be useful to screen for candidate drugs that can overcome MDR. Three-dimensional (3D) cultures have been investigated for their potential to maintain the biological characteristics of cancer cells $[10,11]$, because drug resistance due to efflux activity cannot be modelled effectively using more traditional two-dimensional (2D) monolayer cultures [12]. In a previous study, we demonstrated that HepG2 human hepatic cancer cells formed spheroids on a poly-L-glutamic acid-coated dish and that their doxorubicin (DOX) efflux activity was higher than that of cells cultured in monolayers due to a higher expression of MDR1 [12]. Moreover, the amount of MDR1 per cell in spheroids was comparable to that of hepatic tumour tissue. The half-maximal inhibitory concentration $\left(\mathrm{IC}_{50}\right)$ of DOX was higher in spheroids than in a HepG2 monolayer, whereas both types of culture showed a similar $\mathrm{IC}_{50}$ for 5fluorouracil, which is not exported by MDR1. These results suggest that 3D spheroid hepatic cancer cell cultures provide useful cell-based in vitro assay systems to determine the $\mathrm{IC}_{50}$ values of anticancer agents. However, it can be challenging to prepare spheroids of uniform size under conditions of sufficient medium renewal, which is required for these cell-based in vitro assays [13]. To address this, we investigated whether the MDR of HepG2 cells could be reproduced using Cellbed, a silicate fiber scaffold [14]. Cellbed is commercially available and provides a multi-cell layer-type 3D culture system, in which it is easier to control the HepG2 cell layer size, than in spheroids. We succeeded in identifying greater DOX resistance using Cellbed culture of HepG2 cells than when using a 2D monolayer culture. Furthermore, VRP reduced the $\mathrm{IC}_{50}$ of DOX in the $3 \mathrm{D}$ Cellbed culture, which suggested an in vitro reduction in DOX resistance [15]. However, to explore the efficacy of this assay system for screening agents capable of overcoming $\mathrm{MDR}$, it is important to evaluate their effects on drug resistance in human hepatoma cells in vitro and in vivo. 
Therefore, the present study investigated the effects of VRP on the $\mathrm{IC}_{50}$ of DOX for HepG2 and efflux activity in vitro using 3D Cellbed culture, and on DOX resistance in vivo using xenograft mice models of hepatoma. Furthermore, immunostaining was used to measure MDR1 protein expression in vitro and in vivo.

\section{Materials and Methods}

\section{Cells and culture medium}

Human hepatoma HepG2 cells obtained from the RIKEN Bio Resource Center (Tokyo, Japan) were subcultured as a monolayer in minimum essential medium (Life Technologies, Carlsbad, CA, USA) supplemented with $10 \%$ fetal bovine serum (HyClone, Logan, UT, USA), $100 \mathrm{U} / \mathrm{mL}$ penicillin (Meiji Seika Pharma, Tokyo, Japan), and $100 \mathrm{U} / \mathrm{mL}$ streptomycin (Meiji Seika Pharma, Tokyo, Japan) in a humidified atmosphere of $5 \% \mathrm{CO}_{2}$ and $95 \%$ air at $37^{\circ} \mathrm{C}$.

\section{D culture} Ltd.).

HepG2 cells were cultured using Cellbed ${ }^{\mathrm{TM}}$ (Japan Vilene Company,

\section{Chemicals}

DOX and VRP were obtained from Wako Pure Chemical Industries (Osaka, Japan). DOX was dissolved in sterile water to a stock concentration of $10 \mathrm{mM}$. VRP was dissolved in sterile water at a stock concentration of $200 \mathrm{mM}$.

\section{Assessment of DOX $\mathrm{IC}_{50}$}

The DOX $\mathrm{IC}_{50}$ values for HepG2 cells were determined using the WST-8 [2-(2-methoxy-4-nitrophenyl)-3-(4-nitrophenyl)-5-(2,4disulfophenyl)-2H-tetrazolium, monosodium salt] assay (Cell Counting Kit-8; Dojindo Laboratories, Kumamoto, Japan). For 3D cultures, HepG2 cells $\left(5.0 \times 10^{4} /\right.$ well $)$ were inoculated in Cellbed 96well plates and cultured in a $5 \% \mathrm{CO}_{2}$ humidified incubator at $37^{\circ} \mathrm{C}$ for 7 days. For monolayer cultures, HepG2 cells $\left(2.0 \times 10^{3} /\right.$ well $)$ were inoculated in standard 96-well plates (Sumitomo Bakelite, Tokyo, Japan) for 7 days. Cells were cultured for another $48 \mathrm{~h}$ after adding $\operatorname{DOX}(0.1-300 \mu \mathrm{M})$ in the presence or absence of $100 \mu \mathrm{M}$ VRP. WST-8 solution $(180 \mu \mathrm{L}$ medium and $20 \mu \mathrm{L}$ WST-8 reagent) was added to the plates, followed by incubation for $30 \mathrm{~min}$. Absorbance was measured at a wavelength of $450 \mathrm{~nm}$ on a VersaMax microplate reader (Molecular Devices, Sunnyvale, CA, USA). The DOX $\mathrm{IC}_{50}$ was determined by plotting the logarithm of drug concentration vs. growth rate (percentage of control) of treated cells.

\section{DOX efflux assay}

After growing HepG2 cells for 7 days in Cellbed, the medium was removed and the cells were incubated with fresh medium containing $50 \mu \mathrm{M}$ DOX for $1 \mathrm{~h}$. After the DOX-containing medium was removed, the cells were washed with PBS twice to remove free DOX. The cells were then incubated for $3 \mathrm{~h}$ with medium containing $100 \mu \mathrm{M}$ VRP or medium alone. Aliquots of the conditioned media were collected at 30min intervals and analyzed using a fluorescence spectrophotometer (F-2000, HITACHI, Tokyo, Japan) at excitation and emission wavelengths of $448 \mathrm{~nm}$ and $562 \mathrm{~nm}$ for DOX. In addition, the number of cells in each well was determined using the WST-8 assay.

\section{Assessment of drug resistance in vivo}

The mice were handled in accordance with the guidelines for animal experimentation specified by Japanese law. The animal studies were approved by the Committee on Animal Research of Sojo University. Female NOD/SCID/Jak3 ${ }^{\text {null }}$ (NOJ) mice (5-weeks-old) were provided by Dr. Seiji Okada (Center for AIDS Research, Kumamoto University, Japan) [16]. NOJ mice exhibit a complex type of hyperimmune deficiency; they lack complement and show macrophage and dendritic cell dysfunction (NOD mouse phenotype), they are deficient in mature $\mathrm{T}, \mathrm{B}$, and natural killer cells (Scid mouse phenotype), and show a natural killer cell deficiency derived from the Jak3 ${ }^{\text {null }}$ mouse. HepG2 cells $\left(5.0 \times 10^{6}\right.$ cells) suspended in Matrigel (BD Co., Franklin Lakes, NJ, USA) were subcutaneously inoculated into the dorsal flank of NOJ mice. These animals were randomized into 4 groups: control $(n=9$, physiological saline i.p. and i.v.); VRP-treated group ( $\mathrm{n}=7,25 \mathrm{mg}$ VRP/kg/every 7 days i.p., physiological saline i.v.); DOX-treated group ( $\mathrm{n}=7$, physiological saline i.p., $1 \mathrm{mg} \mathrm{DOX} / \mathrm{kg} /$ every 7 days i.v.); VRP +DOX combination group ( $\mathrm{n}=7,25 \mathrm{mg} \mathrm{VRP} / \mathrm{kg}$ /every 7 days i.p., $1 \mathrm{mg}$ DOX $/ \mathrm{kg} /$ every 7 days i.v.). The doses of DOX and VRP were based on relevant literature [17-19]. Tumor volumes were calculated from (a) the smallest diameter and (b) the longest diameter using the following equation: tumor volume $\left.(\mathrm{V} \mathrm{mm})^{3}\right)=0.5 \times \mathrm{a}^{2} \times \mathrm{b}$. At the end point $(28$ days after cell transplantation), the subcutaneous tumors were removed and weighed.

\section{Hematoxylin and eosin staining and immunostaining}

In vitro specimens of 3D Cellbed cultures were taken on day 9 and in vivo specimens (tumors) were taken from the xenograft mouse model of human hepatoma on day 28. Each specimen was fixed using $10 \%$ formalin solution. The specimens were embedded in paraffin and sectioned $(3 \mu \mathrm{m})$ using a microtome (RM2265; LEICA, Heidelberg, Germany). Sections were stained with hematoxylin and eosin and observed using an optical microscope (Olympus BX-53, Tokyo, Japan). Sections were also dewaxed in xylene and rehydrated through an ethanol series prior to immersion in Tris-EDTA buffer and antigen retrieval by autoclaving at $120^{\circ} \mathrm{C}$ for $20 \mathrm{~min}$. They were then blocked in PBS containing 5\% goat serum for $1 \mathrm{~h}$ at room temperature and washed twice with PBS before incubation with a control IgG (rabbit IgG isotype control, GTX35035; Gene Tex, Irvine, CA, USA) or an anti-MDR1 antibody (rabbit anti-P-glycoprotein antibody, ab170903; Abcam, Cambridge, MA, USA) overnight at $4^{\circ} \mathrm{C}$; these antibodies were diluted 100-fold with PBS containing 3\% BSA. The sections were then washed 3 times with $\mathrm{PBS}$ and endogenous peroxidase activity was blocked by incubation with $3 \% \mathrm{H}_{2} \mathrm{O}_{2}$ solution for 10 min at room temperature. The sections were incubated with a horseradish peroxidase-labeled secondary antibody (goat anti-rabbit IgG antibody, ab6721; Abcam) for $2 \mathrm{~h}$ at room temperature; this antibody was diluted 250 -fold with PBS containing 3\% BSA. The sections were then washed 3 times with PBS. Finally, the signal was detected by incubation with 3,3'-diaminobenzidine tetrahydrochloride. After counterstaining with hematoxylin, ethanol dehydration and xylene substitution were performed, and specimens were prepared for observation by optical microscope using mounting agent (Neo-Mount, 109016, Merck KGaA, Darmstadt, Germany).

\section{Statistical analysis}

Results are presented as mean \pm standard deviation (SD). Data were statistically analysed using Student's t-test. Values of $\mathrm{p}<0.05$ were 
Citation: Inamura K, Emoto K, Ichihara H, Sasaki K, Iwasa T, et al. (2018) Evaluation of an In vitro Approach to the Prediction of In vivo Effects on Multidrug Resistance in Human Hepatoma Cells. J Carcinog Mutagen 9: 318. doi:10.4172/2157-2158.1000318

Page 3 of 5

considered to represent a statistically significant difference (one-sided test).

\section{Results and Discussion}

\section{DOX $\mathrm{IC}_{50}$ in $2 \mathrm{D}$ and $3 \mathrm{D}$ HepG2 cell cultures}

The $\mathrm{IC}_{50}$ of DOX for the growth of HepG2 cells was $5.18 \mu \mathrm{M}$ in $2 \mathrm{D}$ monolayer culture and $55.7 \mu \mathrm{M}$ for 3D Cellbed culture, indicating a higher drug resistance in the latter (Table 1). Furthermore, the $\mathrm{IC}_{50}$ of DOX for 3D-cultured cells was decreased to $1.00 \mu \mathrm{M}$ by treatment with VRP $(100 \mu \mathrm{M})$, which had no effect on cell growth under these experimental conditions; in $2 \mathrm{D}$ culture, the DOX $\mathrm{IC}_{50}$ was reduced to $0.60 \mu \mathrm{M}$ in the presence of VRP (Table 1). These results suggested that the MDR of HepG2 cells and its reversal by VRP was recapitulated in vitro using the 3D Cellbed silicate fiber scaffold [14].

\begin{tabular}{|l|l|}
\hline Culture & DOX IC $\mathbf{C}_{50}(\boldsymbol{\mu M})$ \\
\hline 2D VRP (-) & $5.18 \pm 1.76$ \\
\hline 2D VRP (+) & $0.60 \pm 0.08$ \\
\hline 3D VRP (-) & $55.7 \pm 3.87$ \\
\hline 3D VRP (+) & $1.00 \pm 0.07$ \\
\hline
\end{tabular}

Table 1: Effect of VRP on the DOX $\mathrm{IC}_{50}$ in HepG2 cell cultures HepG2 cells were grown in two-dimensional monolayers (2D) or threedimensional Cellbed cultures (3D). The DOX IC 50 values represent the mean \pm SD of the indicated study groups $(n=3)$, which were cultured in the presence (+) or absence (-) of $100 \mu \mathrm{M}$ VRP.

\section{DOX efflux from 3D-cultured HepG2 cells}

Drug resistance arises due to drug efflux by MDR1 activity [12]. We examined the effects of VRP on DOX efflux from HepG2 cells in 3D Cellbed culture. After pre-incubation of the cells with $50 \mu \mathrm{M}$ DOX at $37^{\circ} \mathrm{C}$ for $1 \mathrm{~h}$, efflux was measured over time in the presence or absence of VRP. After $30 \mathrm{~min}$, DOX efflux was apparently inhibited by VRP, as shown in Figure 1. This result suggests that the reduction of the DOX $\mathrm{IC}_{50}$ in Cellbed culture by VRP reflects VRP-mediated inhibition of MDR1 activity.

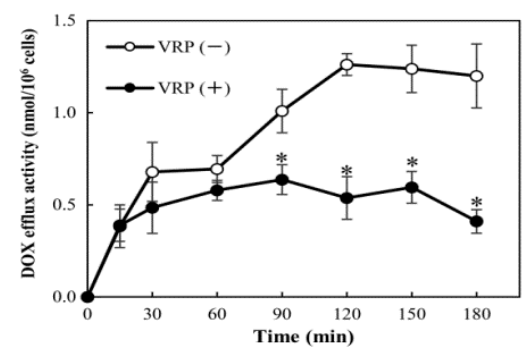

Figure 1: The time course of DOX efflux from HepG2 cells grown in $3 \mathrm{D}$ culture is shown in the presence $(+)$ or absence (-) of VRP. Data represent the mean $\pm \mathrm{SD}$ of the indicated group $(\mathrm{n}=5) ;{ }^{*} \mathrm{p}<0.05$ compared with the VRP (-) group.

\section{Assessment of DOX anti-tumor activity in an in vivo subcutaneous xenograft mouse model of hepatoma}

We initially examined the effects of treatment with DOX $(1 \mathrm{mg} / \mathrm{kg} /$ every 7 days, i.v.) and/or VRP ( $25 \mathrm{mg} / \mathrm{kg} /$ every 7 days, i.p.) on body weight in a xenograft mouse model of hepatoma, generated by subcutaneous inoculation of HepG2 cells in vivo. Body weight decreased gradually in all study groups for 28 days after inoculation; no significant differences were observed between treatment and control groups at the doses tested, indicating that these doses of the compounds showed no severe side effects (Figure 2A).

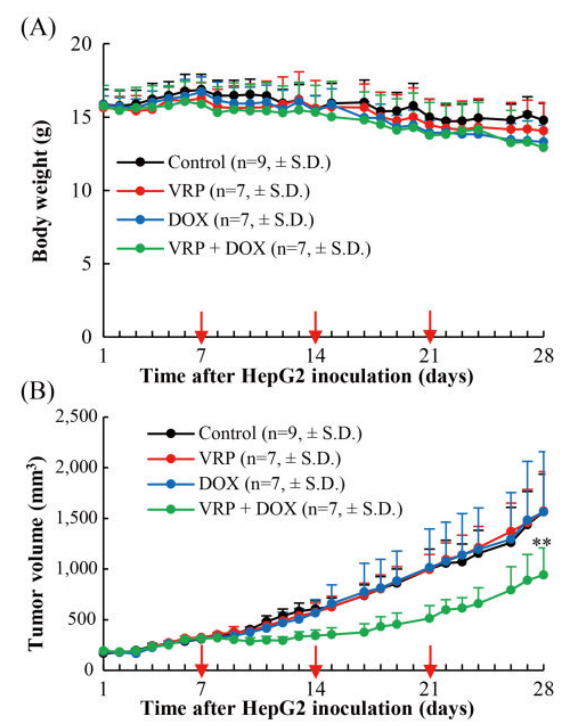

Figure 2: Body weights and tumor growth in the xenograft mouse model of hepatoma. Red arrows show the days when DOX (1 $\mathrm{mg} / \mathrm{kg}$ ) and/or VRP $(25 \mathrm{mg} / \mathrm{kg}$ ) were administered. (A) Mean body weights and (B) Tumor volumes of the indicated study groups. Data represent the mean \pm SD of 7 or 9 animals; ${ }^{* *} \mathrm{p}<0.01$ compared with the other three study groups.

To examine the therapeutic effects of DOX and VRP, we measured tumor volumes in the xenograft mouse model of hepatoma. As shown in Figure 2B, the median tumor volume was $1564.9 \pm 370.5 \mathrm{~mm}^{3}$ in the control group, $1562.6 \pm 594.3 \mathrm{~mm}^{3}$ in the DOX-treated group, and $1571.5 \pm 390.5 \mathrm{~mm}^{3}$ in the VRP-treated group. There were no significant differences between the control, DOX, and VRP treatment groups. However, a marked reduction in tumor volume (942.4 \pm 264.4 $\mathrm{mm}^{3}$ ) was observed in the group treated with both DOX and VRP; this represented a $40 \%$ reduction compared to that in the control group ( $\mathrm{p}<0.01 \mathrm{vs}$. control).

The subcutaneous tumors were resected from the anesthetized mice and weighed. Tumor images are shown in Figure 3A. These indicated that the combination of DOX and VRP produced therapeutic effects on this in vivo xenograft mouse model of hepatoma. Tumors of mice treated with both DOX and VRP were noticeably smaller than those of control, DOX-, or VRP-treated mice. The tumor weights are shown in Figure 3B. Significantly lower tumor weight was observed in mice treated with both DOX and VRP $(1.07 \pm 0.26 \mathrm{~g})$ than in the control group $(1.55 \pm 0.36 \mathrm{~g} ; \mathrm{p}<0.05)$. These results indicate that DOX resistance was ameliorated by VRP in this in vivo model of hepatoma. 
Citation: Inamura K, Emoto K, Ichihara H, Sasaki K, Iwasa T, et al. (2018) Evaluation of an In vitro Approach to the Prediction of In vivo Effects on Multidrug Resistance in Human Hepatoma Cells. J Carcinog Mutagen 9: 318. doi:10.4172/2157-2158.1000318

Page 4 of 5

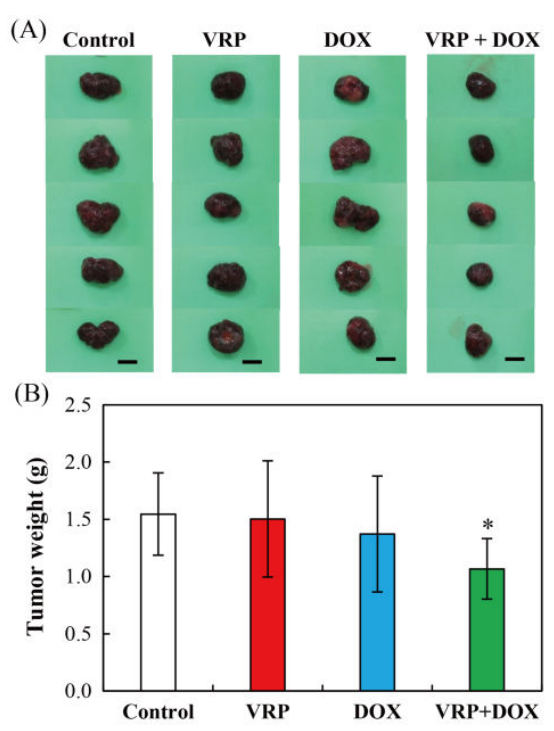

Figure 3: The effects of VRP $(25 \mathrm{mg} / \mathrm{kg})$ on $1 \mathrm{mg} / \mathrm{kg}$ DOX-mediated inhibition of tumor growth in the xenograft mouse model. (A) Photographs of tumors removed on day 28 of the study; a representative five tumors are shown. Scale bars: $1 \mathrm{~cm}$. (B) Weights of tumors removed on day 28 of the study. Data represent the mean \pm SD of 7 or 9 animals; ${ }^{*} \mathrm{p}<0.05$ compared with the control group.

\section{MDR1 expression by 3D-cultured HepG2 cells in vitro and by HepG2 xenografts in vivo}

We evaluated MDR1 expression in 3D Cellbed cultures and in mouse xenografts using immunohistological analyses. The results are shown in Figure 4. Hematoxylin and eosin staining (Figures 4A and $4 \mathrm{E}$ ) indicated that the cells in both specimens were viable and that the Cellbed cultures had formed three or four cell layers by day 9 of culturing (Figure 4A). IgG isotype staining indicated the non-specific background for MDR1 immunostaining in both specimens (Figures $4 \mathrm{~B}$ and 4F). MDR1-positive cells were clearly observed in the 3D Cellbed cultures in vitro and in the xenografted tumors in vivo (Figures $4 \mathrm{C}$ and $4 G)$. Furthermore, MDR1 proteins were partially localized at cell-cell adhesion points (Figures $4 \mathrm{D}$ and $4 \mathrm{H}$ ). Our previous study indicated that MDR1 expression was higher in cells grown as 3D Cellbed cultures than in 2D monolayer cultures [12,15]; this may be related to the development of hypoxia in 3D tissue systems. The hypoxic environment in cancers such as hepatocellular carcinomas upregulates the expression of transcription factors such as hypoxia inducible factor-1 $\alpha$, which regulates MDR1 expression [20,21].

Tissue drug concentrations are determined by the pharmacokinetic properties of the drug after in vivo administration. The evaluation of drug efficacy, therefore, requires comprehensive animal experimentation [22]. The findings of the present study indicated that in relation to drug resistance, the same tendencies were observed in vitro using $3 \mathrm{D}$ Cellbed culture and in vivo using a xenograft mouse model of human hepatoma. These results suggest that $3 \mathrm{D}$ culture of human hepatoma using a silicate fiber scaffold could provide a promising cell-based in vitro assay system for the screening of agents capable of overcoming drug resistance in vivo, before conducting extensive preclinical animal experimentation.

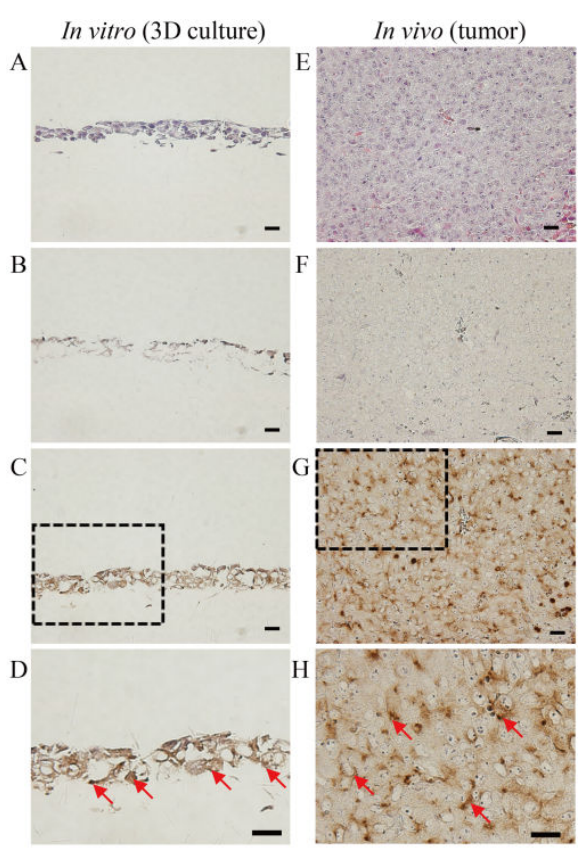

Figure 4: Micrographs showing histology and MDR1 expression of HepG2 cells in 3D Cellbed culture (A-D) and in xenografted HepG2 tumors from the mouse model (E-H). Panels A and E show hematoxylin and eosin staining and panels $\mathrm{B}$ and $\mathrm{F}$ show control IgG isotype staining. MDR1 immunostaining is shown in panels $C$ and $\mathrm{G}$, and at higher magnification in panels $\mathrm{D}$ and $\mathrm{H}$. Scale bars: $20 \mu \mathrm{m}$. Red arrows show expression of MDR1.

\section{Conclusion}

In order to investigate whether a cell-based in vitro assay system could be used to screen for agents capable of overcoming drug resistance in cancer cells, we evaluated the effects of a compound known to reduce resistance (VRP) on the activity and efflux of DOX. These studies were conducted in vitro on $2 \mathrm{D}$ and $3 \mathrm{D}$ cultures of human hepatoma HepG2 cells and in vivo using a HepG2 xenograft mouse model of hepatoma. The DOX $\mathrm{IC}_{50}$ was higher in $3 \mathrm{D}$ Cellbed cultures than in $2 \mathrm{D}$ monolayer cultures, and was reduced by VRP. The drug resistance of HepG2 cells appeared to depend on the MDR1 protein because VRP, which is known to inhibit MDR1 activity, inhibited DOX efflux from HepG2 cells. Furthermore, the administration of combined DOX and VRP in vivo overcame drug resistance in the xenograft mice model of human hepatoma. In addition, MDR1 expression was evaluated in vitro and in vivo using immunostaining. These findings indicate that 3D Cellbed cultures of human hepatoma HepG2 cells could provide a promising cell-based in vitro assay system for the screening of agents with the potential to overcome hepatic cancer cell drug resistance in vivo.

\section{Acknowledgements}

This study was supported in part by a Grant-in-Aid for Scientific Research from the Ministry of Education, Culture, Sports, Science, and Technology of Japan (no. 26420804) and the Research on Development 
Citation: Inamura K, Emoto K, Ichihara H, Sasaki K, Iwasa T, et al. (2018) Evaluation of an In vitro Approach to the Prediction of In vivo Effects on Multidrug Resistance in Human Hepatoma Cells. J Carcinog Mutagen 9: 318. doi:10.4172/2157-2158.1000318

Page 5 of 5

of New Drugs from Japan Agency for Medical Research and Development, AMED (no.17ak0101073j0001).

\section{References}

1. Gottesman M, Pastan I (1993) Biochemistry of multidrug resistance mediated by the multidrug transporter. Annu Rev Biochem 62: 385-427.

2. Dean M, Hamon Y, Chimini G (2001) The human ATP-binding cassette (ABC) transporter superfamily. J Lipid Res 42: 1007-1017.

3. Tatsuta T, Naito M, Oh-hara T, Sugawara I, Tsuruo T (1992) Functional involvement of P-glycoprotein in blood-brain barrier. J Biol Chem 267: 20383-20391.

4. Tsuruo T, Naito M, Tomida A, Fujita N, Mashima T, et al. (2003) Molecular targeting therapy of cancer: drug resistance, apoptosis and survival signal. Cancer Sci 94: 15-21.

5. Tsuruo T, Iida H, Tsukagoshi S, Sakurai Y (1981) Overcoming of vincristine resistance in P388 leukemia in vivo and in vitro through enhanced cytotoxicity of vincristine and vinblastine by verapamil. Cancer Res 41: 1967-1972.

6. Watanabe T, Kokubu N, Charnick SB, Naito M, Tsuruo T, et al. (1997) Interaction of cyclosporin derivatives with the ATPase activity of human P-glycoprotein. Br J Pharmacol 122: 241-248

7. Yusa K, Tsuruo T (1989) Reversal mechanism of multidrug resistance by verapamil: direct binding of verapamil to P-glycoprotein on specific sites and transport of verapamil outward across the plasma membrane of K562/ADM cells. Cancer Res 49: 5002-5006.

8. Naito M, Oh-hara T, Yamazaki A, Danki T, Tsuruo T (1992) Reversal of resistance by an immunosuppressive agent FK-506. Cancer Chemother Pharmacol 29: 195-200.

9. Tsuruo T, Iida H, Tsukagoshi S, Sakurai Y (1982) Increased accumulation of vincristine and adriamycin in drug-resistant P388 tumor cells following incubation with calcium antagonists and calmodulin inhibitors. Cancer Res 42: 4730-4733.

10. Kabsch W, Vandekerckhove J (1992) Structure and function of actin. Annu Rev Biophys Biomol Struct 21: 49-76.

11. Langhans SA (2018) Three-dimensional in vitro cell culture models in drug discovery and drug repositioning. Front Pharmacol 9: 1-14.
12. Oshikata A, Matsushita T, Ueoka R (2011) Enhancement of drug efflux activity via MDR1 protein by spheroid culture of human hepatic cancer cells. J Biosci Bioeng 111: 590-593.

13. Sakai S, Inamoto K, Liu Y, Tanaka S, Arii S, et al. (2012) Multicellular tumor spheroid formation in duplex microcapsules for analysis of chemosensitivity. Cancer Sci 103: 549-554.

14. Yamaguchi Y, Deng D, Sato Y, Hou YT, Watanabe R, et al. (2013) Silicate fiber-based 3D cell culture system for anticancer drug screening. Anticancer Res 33: 5301-5310.

15. Mizutami T, Ohta Y, Nakamura M, Komizu Y, Iwasa T, et al. (2017) Induction of drug resistance in human hepatoma cells cultured on a silicate fiber-based 3D scaffold. Adv Biochem Biotechnol 2017: 112.

16. Okada S, Harada H, Ito T, Saito T, Suzu S (2008) Early development of human hematopoietic and acquired immune systems in new born NOD/ Scid/ Jak3null mice intrahepatic engrafted with cord blood-derived CD34+ cells. Int J Hematol 88: 476-482.

17. Fan C, Zheng W, Fu X, Li X, Wong YS, et al. (2014) Strategy to enhance the therapeutic effect of doxorubicin in human hepatocelluar carcinoma by selenocystine, a synergistic agent that regulates the ROS-mediated signaling. Oncotarget 5: 2853-2863.

18. Patel KJ, Tannock IF (2009) The influence of P-glycoprotein expression and its inhibitors on the distribution of doxorubicin in breast tumors. BMC Cancer 9: 356.

19. Osman AMM, Mohamad MA, Abdel-Wahab AHA, Sayed-Ahmad MM (2000) Modulation by verapamil of doxorubicin induced expression of multidrug resistance gene (MDR1) in murine tumor cells. J Egypt Natl Canc Inst 12: 221-227.

20. Comerford KC, Wallace TJ, Karhausen J, Louis NA, Montalto MC, et al. (2002) Hypoxia-inducible factor-1-dependent regulation of multidrug resistance (MDR1) gene. Cancer Res 62: 3387-3394.

21. Wartenberg M, Ling FC, Müschen M, Klein F, Acker H, et al. (2003) Regulation of the multidrug resistance transporter P-glycoprotein in multicellular tumor spheroids by hypoxia-inducible factor-1 (HIF-1) and reactive oxygen species. FASEB J 17: 503-505.

22. Chun YJ (2014) Three dimensional in vitro human tissue models for ADME-Tox studies. Drug Metab Pharmacokinet 29: 431-432. 